\title{
Advances in the Hyphenation of Flow Analysis Techniques with Liquid Separations for Pharmaceutical Analysis
}

\author{
Anastasios Economou* \\ Department of Chemistry, National and Kapodistrian University of Athens, Athens 157 71, Greece
}

\section{COMMENTARY}

This commentary critically discusses the main advances in the field of flow analysis techniques hyphenated with liquid separations for pharmaceutical analysis over the last 15 years. Flow analysis techniques provide a convenient way to perform chemical assays with significant benefits in terms of rapidity, simplicity and cost of instrumentation, economy, precision, versatility in sample handling and potential for automation. The first-generation flow injection analysis (FIA), developed in the 1970's, has been supplemented by a host of second-generation and third-generation flow methods developed in the 1990's and 2000's such as sequential injection analysis (SIA) and multi-syringe flow injection analysis (MSFIA) [1]. However, the main drawback of these techniques is their limited ability to simultaneously determine several analytes. Therefore, the hyphenation of flow techniques with separation techniques is a very attractive because it enables multi-component analysis. It was the introduction of monolithic columns [2] that has made possible the direct coupling of flow techniques with liquid separations platforms and has led to the development of hyphenated approaches such as FIC, SIC and MSC [3-5]. In all these techniques, a separation column is inserted in the flow path between the point of sample introduction and the detector; the main principles of operation can be found in the relevant literature [3-7]. FIC, SIC and MSC offer distinct advantages in terms of rapidity, simplicity, versatility and capital costs compared with high performance liquid chromatography (HPLC) and have been applied to the determination of a large number of pharmaceuticals [3-5]. When simple mixtures are considered, these hyphenated methods produce results that are comparable to HPLC [8,9]. The main advances in the hyphenation of flow analysis techniques with liquid separations for the purposes of pharmaceutical analysis are identified below.

\section{From low-pressure to medium-pressure separations}

The choice and matching of the appropriate components is an important prerequisite for coupling flow analysis techniques to separation columns. Traditional flow analysis techniques normally operate at low pressures ( $<100 \mathrm{psi})$ and, therefore, pressure problems are rarely encountered. Since the introduction of a separation column in the flow manifold causes an increase in the backpressure, the most critical aspect is the pressure specifications of the components to be used. FIC makes use of peristaltic pumps or the low-pressure variant of version of the MilliGAT pump which are limited to backpressure of around 100 psi [10-12]. Similarly MSC systems make use of low-pressure solenoid valves that also pose some limitations on the pressure that could be achieved [13]. Early work in SIC typically made use of medium-pressure syringe pumps which can withstand substantially higher back pressures (up to $500-700 \mathrm{psi}$ ) such as the commercial discontinued FIAlab® 3000 analyzer (which was modified in-house for SIC applications) which was superseded by the dedicated (also discontinued) SIChrom ${ }^{\mathrm{TM}}$ system (FIAlab Instruments, USA) [14]. Even higher pressures (up to $2000 \mathrm{psi}$ ) can be achieved with industrial syringe pumps recently introduced in SIC [15] or the new high-pressure version of the MilliGAT pump (rated at 1500 psi) as an alternative option [16]. Of course, the use of higher pressures also poses limitations on the valves used in the manifold; for instance the low-pressure variants of the widely used the Vivi-Valco injection and selection valves can withstand only up to $250 \mathrm{psi}$ and switching to a higher pressure system would inevitable require a change of the valve to one rated for higher pressures (e.g. suitable for HPLC tolerant to 5000 psi). This ultimate development of such a medium-pressure system is typified by the current commercial SIChromII ${ }^{\mathrm{TM}}$ system (FIAlab Instruments, USA) [17]. The ability of the manifold to handle higher pressures affords significant advantages as it enables the use of potentially longer and more densely packed columns (to enhance the separation efficiency) and higher flow rates (to speed up the analysis). However, high-pressure components involve substantially higher purchasing costs and one has to consider the relative benefitto-cost ratio with regards to the intended application.

\section{Separation columns}

The backpressure limitations discussed above, especially in relation to the early-generation low-pressure systems, also impose some limitations in terms of the type and length of the separation column. Therefore, initially applications were limited to C18 reverse-phase monolithic columns, typically $5-25 \mathrm{~mm}$ long. The introduction of medium-pressure systems allowed the use of longer (e.g. $50 \mathrm{~mm}$ ) monolithic columns [18]. More recently, short fused-core columns

"Correspondence to: Anastasios Economou, Department of Chemistry, National and Kapodistrian University of Athens, Athens 157 71, Greece, Tel: +302107274208; Fax: +302107274750; E-mail: aeconomo@chem.uoa.gr

Received: Febrruary 09, 2021; Accepted: February 17, 2021; Published: February 24, 2021

Citation: Economou A (2019) Advances in the Hyphenation of Flow Analysis Techniques with Liquid Separations for Pharmaceutical Analysiss. Pharm Anal Acta 10:613. doi: $10.35248 / 2153-2435.19 .10 .613$

Copyright: (C) 2019Economou A. This is an open-access article distributed under the terms of the Creative Commons Attribution License, which permits unrestricted use, distribution, and reproduction in any medium, provided the original author and source are credited. 
with dimensions 5-30 $\mathrm{mm}$ have been successfully implemented in medium-pressure chromatographic systems $[19,20]$. In addition, other packings (such as a cyano $5 \mathrm{~mm}$ long monolithic column for sub-1 min separation of dues in pharmaceuticals) [21] and RPamide, F5 and phenyl-hexyl materials [19] have been introduced. An interesting approach is the use of two-columns of different length, each operating in an isocratic separation mode but with different compositions of mobile phase [22].

\section{Development of gradient elution protocols}

The majority of hyphenated flow analysis-separations systems in the literature make use of isocratic elution protocols. However, when the target analytes exhibit a wide variation in polarity, isocratic elution becomes inefficient. A step change of the eluent is the most widely applicable and easiest method to modulate the elution force of the mobile phase and involves sequential elution of the analytes with two or more separate solvents of different polarity [23,24]. Another alternative is the generation of a gradient profile in the holding coil of a SIC manifold by aspirating zones of different solvents with different polarities; the stacked zones are then directed to the column and cause the elution of the analytes in order of varying polarity [25]. Obviously, in this case the gradient profile is not steplike since the zones of the solvents undergo mutual dispersion and mixing. Recently, our group proposed a powerful method for the generation of a strictly linear two-solvent gradient elution profile by appropriate flow rate modulation of 2 pumps delivering solvent of different polarities [11].

\section{On-line sample preatreatment}

One of the main advantages of flow analysis methods is the exploitation of zone fluidics for on-line sample pretreatment. Essentially the same protocols can be adapted to hyphenated SIC and MSC systems for simple filtration and dilution [26] or more complicated operations such as solid-phase extraction using packed minicolumns [27-29] or the head of the separation column [30].

\section{Automation and computer control}

FIC manifolds with isocratic elution (which involve continuous and unidirectional flow of the mobile flow) do not normally require the use of computer control since both the pump and the injection valve can be manually controlled [12]. In this sense, these systems are uncomplicated, relatively inexpensive, simple to operate and also suitable for educational use but, on the other hand, their potential for automation is limited. Of course, when complex gradient elution protocols are required in FIC, the use of computercontrolled pumps and valves, as well as the relevant software, becomes necessary, resulting in fully automated FIC manifolds [11]. The introduction of SIC and MSC has also necessitated the use of computed control for solution handling; the controlling computed can also exploited for data acquisition. This trend has resulted in the development of hyphenated chromatography systems using commercial [17] or proprietary control and data acquisition software $[11,13]$.

To summarize, in this commentary the key advances, advantages and limitations of FIC, MSC and SIC have been identified and the author's opinion that hyphenation of flow analysis techniques with liquid separations offers wide scope in pharmaceutical analysis applications and these systems can serve successfully as complimentary to HPLC for both separation and quantification purposes.

\section{REFERENCES}

1. Melchert WR, Reis BF, Rocha FRP. Green chemistry and the evolution of flow analysis. A review Anal Chim Acta. 2012;714:8-19.

2. Gama MR, Rocha FRP, Bottoli CBG. Monoliths: Synthetic routes, functionalization and innovative analytical applications. Trends Anal Chem. 2019;115:39-51.

3. Idris AM. The second five years of sequential injection chromatography: significant developments in the technology and methodologies. Crit Rev Anal Chem. 2014;44:220-32.

4. Fernández M, Forteza R, Cerdà V. Monolithic columns in flow analysis: a review of SIC and MSC techniques. Instrum Sci Tech. 2012;40:90-99.

5. Hartwell SK, Kehling A, Lapanantnoppakhun S, Grudpan K. Flow injection/sequential injection chromatography: a review of recent developments in low pressure with high performance chemical separation. Anal Lett. 2013;46:1640-1671.

6. Fernández M, Miró M, González HM, Cerdà V. Modulation of mobile phase composition in flow-injection/sequential-injection chromatography exploiting multisyringe flow analysis. Anal Bioanal Chem. 2008;391:817-25.

7. Chocholous P, Solich P, Satinsky D. An overview of sequential injection chromatography. Anal Chim Acta. 2007;600:129-35.

8. Idris AM, Alnajjar AO. Optimizing SIC Assay Method for Acetyl Salicylic Acid and Rosuvastatin and Adapting to HPLC with Performance Comparison. Acta Chromatograph. 2015;27:111-25.

9. Idris AM, Elgorashe, REE. Sequential injection chromatography against HPLC and CE: Application to separation and quantification of amoxicillin and clavulanic acid. Microchem J. 2011;99:174-179.

10. Ballesta Claver J, Valencia MC, Capitan-Vallvey LF. Analysis of parabens in cosmetics by low pressure liquid chromatography with monolithic column and chemiluminescent detection. Talanta. 2009;79:499-506.

11. Barbatsi M, Koupparis M, Economou A. A new flow-injection chromatography method exploiting linear-gradient elution for fast quantitative screening of parabens in cosmetics. Anal Methods. 2016;8:8337-8344

12.Santos IC, Mesquita RBR, Amorim CL, Castro PML, Rangel AOSS Development of a low pressure chromatographic flow system for monitoring the biodegradation of ofloxacin and ciprofloxacin. Anal Methods. 2016;8:5457-5465.

13.González-San Miguel HM, Alpízar-Lorenzo JM, Cerdà-Martín, V. Development of a new high performance low pressure chromatographic system using a multisyringe burette coupled to a chromatographic monolithic column. Talanta. 2007;72:296-300.

14.Šatínský D, DosSantos LML, Sklenářová $H$, Solich $P$, Conceição M, Montenegro BSM, et al. Sequential injection chromatographic determination of ambroxol hydrochloride and doxycycline in pharmaceutical preparations. Talanta. 2005;68:214-218.

15.Chocholouš P, Šatinsky D, Solich P. New generation of sequential injection chromatography: Great enhancement of capabilities of separation using flow analysis. Talanta. 2019;204:272-277.

16.https://www.vici.com/liqhand/m6.php

17. https://www.flowinjection.com/hardware/sia-analyzers/fialab-sichrom

18. Idris AM. Sequential injection chromatography forbiofluidic analysis: application to promethazine assay. J Liquid Chromatogr Rel Tech. 2012;35:2884-2899.

19. Batista AD, Rocha FRP. A flow injection low-pressure chromatographic system exploiting fused-core columns. Anal Methods. 2014;6:9299. 9304. 
20.Chocholous P, Kosarova L, Satinsky D, Sklenarova H, Solich P. Enhanced capabilities of separation in Sequential Injection Chromatography-Fused-core particle column and its comparison with narrow-bore monolithic column. Talanta. 2011;85:1129-34.

21. Davletbaeva P, Chocholouš P, Bulatov A, Šatínský D, Solich P. Sub-1 min separation in sequential injection chromatography for determination of synthetic water-soluble dyes in pharmaceutical formulation. J Pharm Biomed Anal. 2017;143:123-129.

22. Chocholous P, Satinsky D, Sklenarova H, Solich P. Two-column Sequential Injection Chromatography-New approach for fast and effective analysis and its comparison with gradient elution chromatography. Anal Chim Acta. 2010;668:61-66.

23. Rodasa Lindomar M, Portugal A, Avivar J, Estela JM, Cerdà V. Parabens determination in cosmetic and personal care products exploiting a multi-syringe chromatographic (MSC) system and chemiluminescent detection. Talanta. 2015;143:254-262.

24.García-Jiménez JF, Valencia MC, Capitán-Vallvey LF. Parabens determination with a hybrid FIA/HPLC system with ultra-short monolithic column. J Anal Chem. 2010;65:188-194.

25.Koblova P, Sklenarova H, Chocholous P, Polasek M, Solich P. Simple automated generation of gradient elution conditions in sequential Injection chromatography using monolithic column. Talanta. 2011;84:1273-1277.
26.Zacharis CK, Verdoukas A, Tzanavaras PD, Themelis DG. Automated sample preparation coupled to sequential injection chromatography: On-line filtration and dilution protocols prior to separation. J Pharm Biomed Anal. 2009;49:726-732.

27. Šrámková I, Chocholouš P, Sklenářová H, Šatínský D. On-line coupling of Micro-Extraction by Packed Sorbent with Sequential Injection Chromatography system for direct extraction and determination of betaxolol in human urine. Talanta. 2015;143:132-137.

28. Maya JF, Estela M, Cerdà V. Interfacing on-line solid phase extraction with monolithic column multisyringe chromatography and chemiluminescence detection: An effective tool for fast, sensitive and selective determination of thiazide diuretics. Talanta. 2010;80:1333 1340 .

29. Obando MA, Estela JM, Cerdà V. Multi-syringe chromatography (MSC) system for the on-line solid-phase extraction and determination of hydrochlorothiazide and losartan potassium in superficial water, groundwater and wastewater outlet samples. J Pharm Biomed Anal. 2008;48:212-217.

30.Batista AD, Rocha FRP. On-column preconcentration in sequential injection chromatography: application to determination of parabens. Anal Methods. 2015;7:4371-4375. 